BULL. AUSTRAL. MATH. SOC.

\title{
A DENSITY PROBLEM FOR HARDY SPACES OF ALMOST PERIODIC FUNCTIONS
}

\author{
ROBYN OWENS
}

\begin{abstract}
We construct a counterexample, for $p=1$, to the conjecture posed by Milaszevitch in 1970: is the space of functions which are analytic in the upper half plane and uniformly almost periodic in its closure dense in the Hardy space $H^{p}(0<p<\infty)$ of analytic almost periodic functions?
\end{abstract}

\section{Introduction}

Let $A_{0}$ denote the space of functions that are analytic in the open upper half plane and uniformly almost periodic in its closure. In this paper we construct an analytic function $f$ which is uniformly almost periodic on any horizontal line in the open half plane in such a way that. $f$ is a member of the Hardy space $H^{l}$ of almost periodic functions and yet $f$ does not belong to the closure of $A_{0}$ in $H^{\perp}$. This provides a. counterexample to the conjecture, which we shall refer to as the density problem, posed by Milaszevich [4] in 1970: is $A_{0}$ dense in $H^{p}$, $0<p<\infty$ (in analogy with the classical case where the space of bounded analytic functions on the unit disc $D$ is dense in $\left.H^{p}(D)\right)$ ?

Received 29 November 1984. The author would like to thank N.Th. Varopoulos and Walter Bloom for their kind help and encouragement.

Copyright Clearance Centre, Inc. Serial-fee code: 0004-9727/84 $\$$ A2.00 +0.00 . 
The class of Hardy spaces $H^{p}$ of almost periodic functions combines many of the properties of the classical Hardy spaces of the upper half plane and the unit disc. $H^{p}$ consists of functions that are analytic in the upper half plane, uniformly almost periodic on any horizontal line, and yet constrained by a bounded measure on the boundary $\mathbb{R}$ of the upper half plane. This measure axises naturally [2] in the theory of abstract harmonic analysis, where the unit circle is replaced by any locally compact abelian group $G$ and the set of indices over which one forms a "trigonometric series" is taken to be the dual group of $G$. In our case, the group $R$ of real numbers endowed with the discrete topology is considered; its dual group can then be identified as blR., the Bohr compactification of $\mathbb{R}$. The natural measure which arises by considering the space of generalized analytic functions on $b \mathbb{R}$ [2] turns out to define precisely the condition that the space be an amalgam of $L^{p}$ and $Z^{q}$ of $\mathbb{R}$ [3].

\section{Notation and preliminary results}

We denote by $P$ the open upper half plane and by $\bar{P}$ its closure. The unit disc is denoted $D$ and its boundary is $\partial D=\mathbb{T}$, the unit circle in the complex plane $\mathbb{C}$. Unless otherwise indicated, ${ }_{L}^{p}$ spaces on subsets of the complex plane are taken with respect to Lebesgue measure on the appropriate subset.

$$
\begin{aligned}
\text { Suppose } 0 \leq & a<b \leq \infty \text { and define } \\
& \operatorname{strip}(a, b)=\{x+i y \in \mathbf{C}: a<y<b\} .
\end{aligned}
$$

If $f$ is an harmonic function defined on $P$ was say $f$ is uniformly almost periodic in the strip $(a, b)$ if for any $\varepsilon>0$ there is a $T>0$ such that any real interval of length $T$ contains $\tau$ satisfying

$$
|f(z+\tau)-f(z)|<\varepsilon, z \in \operatorname{strip}(a, b) .
$$

The above definitions can be extended in the obvious way to half open and closed strips in $P$.

Given a function $f$ on $\mathbb{C}$ we define the translated and reflected function $f_{v}$ by

$$
f_{v}(x+i y)=f(v-x+i y), \quad v \in \mathbb{R}
$$


Let $0<p<\infty$ and consider the collection of all analytic functions $f$ on $P$ which satisfy the following two conditions:

(2.1) for any $\varepsilon>0, f$ is uniformly almost periodic in the strip $[\varepsilon, \infty) ;$

(2.2) $\|f\|_{p}^{p}=\sup _{y>0} \sup _{v \in \mathbb{R}} \frac{1}{\pi} \int_{-\infty}^{\infty}|f(x+i y)|^{p} \frac{d x}{1+(v-x)^{2}}<\infty$.

We define the Hardy space $H^{\mathscr{P}}$ as the space of all analytic functions on $P$ satisfying (2.I) and (2.2).

When $p \geq 1$ the quantity $\|f\|_{p}$ defines a norm under which the class of functions $H^{p}$ is a Banach space and it is shown in [2] that these spaces display many of the characteristics of the classical Hardy spaces on the disc and the upper half plane. In particular, if $f \in H^{p}$ and $1 \leq p<\infty$ then the boundary function

$$
f(x)=\lim _{y \rightarrow 0} f(x+i y)
$$

exists almost everywhere and $f(x+i y)$ can be reproduced as the Poisson integral of $f(x)$. The boundary function $f$ satisfies

$$
\sup _{v \in \mathbb{R}} \int_{-\infty}^{\infty}|f(x)|^{p} \frac{d x}{1+(v-x)^{2}}<\infty \text {, }
$$

a fact which can also be seen directly using Fatou's Lemma. In terms of the translated and reflected function $f_{v}$ we have

$$
\sup _{v \in R} \int_{-\infty}^{\infty}|f(x)|^{p} \frac{d x}{1+(v-x)^{2}}=\sup _{v \in R} \int_{-\infty}^{\infty}\left|f_{v}(x)\right|^{p} \frac{d x}{1+x^{2}}
$$

Let $P^{(y)}(t)=y / \pi\left(y^{2}+t^{2}\right), y, t \in \mathbf{R}$, denote the Poisson kernel and let $\chi_{I}$ denote the characteristic function of a set $I \subseteq R$. It is shown in [1] that there exist constants $C_{1}$ and $C_{2}$ such that

$$
C_{\perp} \sup _{v \in R} \int_{v}^{v+1}|f(x)|^{p} d x \leq \sup _{v \in R} \int_{-\infty}^{\infty}|f(x)|^{p} \frac{d x}{1+(v-x)^{2}}
$$




$$
\leqslant c_{2} \sup _{v \in \mathbb{R}} \int_{v}^{v+1}|f(x)|^{p} d x,
$$

so that by replacing $p^{(1)}(v-t)$ with the box kernel $x_{[v, v+1]}$, the condition (2.3) becomes equivalent to the condition

$$
\sup _{v \in R} \int_{v}^{v+1}|f(x)|^{p} d x<\infty .
$$

Condition (2.4) is usually expressed [3] by saying that $f$ belongs to the amalgam space $\left(L^{p}, Z^{\infty}\right)$, with the quantity on the left hand side of (2.4) denoted by $\|f\|_{p, \infty}^{p}$.

When $u(x+i y)$ is an harmonic function in $P$ satisfying the condition (2.2) and $p \geq 1$, the conjugate function $\tilde{u}$ can be computed explicitly by defining the Hilbert transform on its boundary function $u(x)$. To do this we use the conformal mapping $\psi$ between the unit disc $D$ and the upper half plane given by

$$
\psi(z)=-i\left[\frac{z-1}{z+1}\right], \quad z \in \bar{D}, \quad z \neq-1
$$

Via this mapping we can define the Hilbert transform $H u_{v}$ of each of the functions $u_{v}(x)=u(v-x)$, since each $u_{v}$ belonging to ${ }_{L}^{P}\left(\mathbb{R} ; d x /\left(1+x^{2}\right)\right)$ is mapped onto a function $u_{v} \circ \psi$ belonging to ${ }_{L}^{P}(\mathbf{T})$ and the usual Hilbert transform $H$ is well-defined on this space. Explicitly, $H u_{v}$ is defined as $H\left(u_{v} \circ \psi\right) \circ \psi^{-1}$. Under the conformal mapping $\psi$ however, the translations of $u$ are not preserved; that is, in general $H u_{v} \neq(H u)_{v}$. But since (the Poisson integrals of) $H u_{v}$ and $\left(\mathrm{Hu}_{v}\right.$ both represent the imaginary part of the same analytic function their difference can only be a constant; we write $H u_{v}-(H u)_{v}=c(u, v)$.

Now if $u(x)$ is a continuous bounded function on $\mathbb{R}$ then $\tilde{u}(x)$ is defined by the Hilbert transform and, up to an additive constant, is the boundary function of the conjugate of the harmonic extension of $u$. It is known [1] that $u \mapsto \tilde{u}$ is a continuous function from $L^{\infty}$ into BMO, the 
space of functions of bounded mean oscillation.

PROPOSITION 1. If $u$ is a continuous periodic function on $R$ then $\tilde{u}$ is also periodic.

Proof. Without loss of generality we suppose $u$ to be $2 \pi$-periodic. Then $u$ can be identified with a continuous function $g$ defined on $T$ and $g$ has a Fourier series expansion

$$
g(\theta) \sim \sum_{n=-\infty}^{\infty} a_{n} e^{i r i \hat{\theta}}, 0 \leq \theta<2 \pi .
$$

The conjugate function $\tilde{g}$ is the unique harmonic function satisfying $g+i \tilde{g}$ analytic in $D$ and $\tilde{g}(0)=0$. It is well known that $\tilde{g}$ has a Fourier series

$$
-i \sum_{n \neq 0} \operatorname{sgn} n a_{n} e^{i n \theta}
$$

in the sense that the conjugate Poisson integral of $g$ has an expression of the form

$$
\tilde{g}\left(\mathrm{re}^{i \theta}\right)=-i \sum_{n \neq 0} \operatorname{sgn} n a_{n}{ }^{|n|} e^{i n \theta}
$$

and this tends to a limit almost everywhere on $\pi$ as $r \uparrow 1$.

Now let us define the function $\tilde{u}=\tilde{g} \circ \phi$ on $P$ where $\phi(z)=\exp (i z)$. Since $\phi$ is analytic, $\tilde{u}$ is harmonic and periodic on $P$. In the limit

$$
\tilde{u}(t)=-i \sum_{n \neq 0} \operatorname{sgn} n a_{n} e^{i n t}, t \in \mathbf{R},
$$

defines almost everywhere a periodic function on $R$ and gives a conjugate function for $u$ since

$$
(u+i \tilde{u})(z)=2 \sum_{n \geq 0} a_{n} e^{i n z}
$$

is analytic in $P$. This completes the proof.

We denote by $\Gamma(t)$ the cone

$$
\Gamma(t)=\{z=x+i y \in P:|x-t|<y\}, t \in \mathbf{R} .
$$

If $u$ is an harmonic function on $P$ we define the non-tangential maximal 
fronction $u^{*}$ of $u$ by

$$
u^{*}(t)=\sup _{z \in \Gamma(t)}|u(z)|, t \in \mathbb{R} \text {. }
$$

The following proposition is proved in [5] and although the result is important for the construction of the counterexample in $\$ 3$, the techniques of its proof are not and so the proof is omitted.

PROPOSITION 2. Suppose $u$ is harmonic in $P$ and $u^{*} \in\left(L^{1}, l^{\infty}\right)$. If $x \rightarrow \tilde{u}(x+i y) \in L^{\infty}$ for some $y>0$, then $\tilde{u} \in\left(L^{1}, z^{\infty}\right)$.

Note that in this case we have $u+i \tilde{u} \in H^{l}$, for $u^{*}$ dominates $u$ and the norm condition for membership of $\left(L^{I}, z^{\infty}\right)$ is precisely that of belonging to $H^{1}$.

A partial answer to the density problem was given by Milaszevitch [4, p. 425] in 1970 and may be stated as follows.

PROPOSITION 3. Let $f \in H^{p}, 0<p<\infty$. If $f$ belongs to the closure of $A_{0}$ in $H^{p}$.then the function $t \rightarrow f_{t}$ is continuous as a finction from $\mathbb{R}$ into $H^{p}$.

Recall that $f_{t}$ denotes the function $f$ translated and reflected in its real variable by $t \in \mathbb{R}$.

We now proceed to construct a function $f \in H^{I}$ that does not satisfy the above continuity condition.

\section{The construction}

The idea behind our counterexample is as follows:- we shall construct a real-valued bounded function $u \in\left(L^{l}, Z^{\infty}\right)$ such that the function $t \rightarrow u_{t}$ is not continuous from $R$ into $\left(L^{l}, z^{\infty}\right)$. The function $u$ is to be such that its harmonic extension is uniformly almost periodic on any line $L_{y}=\{\operatorname{Im} z=y\}, y>0$, and also that the harmonic extension of its conjugate $\tilde{u}$ is uniformly almost periodic on any such line. The maximal function $u^{*}$ will belong to $\left(L^{1}, l^{\infty}\right)$ and since $\tilde{u}$ is bounded on 
any line $L_{y}, y>0$ (being uniformly almost periodic there), this implies $u \in\left(L^{l}, l^{\infty}\right)$, by Proposition 2. Thus $f=u+i \tilde{u} \in H^{1}$. But as $t \rightarrow f_{t}$ is not continuous as a function from $\mathbb{R}$ into $H^{1}$ we will have by Proposition 3 that $f$ does not belong to the closure of $A_{0}$ in $H^{\mathcal{I}}$.

To begin, take a periodic progression

$$
n_{k}^{(1)}=k T_{1}, k \in \mathbb{Z},
$$

where $T_{1} \geq 10$ is a large integer to be determined later. Let $\rho$ be a $C^{\infty}$ function with $\left\|\rho^{\prime}\right\|_{\infty}<\infty$ and such that $\rho \equiv 1$ on $\left[\frac{1}{4}, \frac{3}{4}\right], \rho \equiv 0$ outside $[0,1]$, and $\rho$ is monotonic on each of the intervals $\left[0, \frac{1}{4}\right]$ and $\left[\frac{3}{4}, 1\right]$. Define $\rho_{1}$ periodically by $\rho_{1}(x)=\rho(x)$, for $x \in\left[0, T_{1}\right]$ and $\rho_{1}\left(x+T_{1}\right)=\rho_{1}(x)$, for $x \in \mathbb{R}$. The function $u_{1}$ is then determined by the formula.

$$
u_{1}(t)=\rho_{1}(t) \sin \left(2 \pi N_{1} t\right), \quad t \in \mathbb{R},
$$

where $N_{1}$ is a large integer to be determined later. Note that $u_{1}$ is periodic with period $T_{1}$ and so its harmonic extension is also periodic.

Proceeding in this fashion, suppose we have defined $u_{1}, u_{2}, \ldots, u_{m-1}$. To define $u_{m}$ we begin by defining the arithmetic progression

$$
n_{k}^{(m)}=a_{m}+k T_{m}, k \in \mathbb{Z}
$$

where $a_{m} \in \mathbb{Z}$ satisfies

$$
a_{m} \in \bigcap_{i<m}^{\infty} \bigcup_{j=1}^{\infty}\left[\frac{{ }^{4} j T_{i}}{10}, \frac{6 j T_{i}}{10}\right]
$$

and $T_{m} \geq 10 T_{m-1}$ is a large integer to be determined later.

Note that the condition $T_{k} \geq 10 . T_{k-1}$ for each $k$ ensures that it is possible to choose each $a_{m}$, that is, 


$$
\bigcap_{i<m} \bigcup_{j=1}^{\infty}\left[\frac{4 j T_{i}}{10}, \frac{6 j T_{i}}{10}\right] \neq \varnothing .
$$

Define $\rho_{m}$ periodically by $\rho_{m}(x)=\rho\left(x-a_{m}\right)$ for $x \in\left[a_{m}, a_{m}+T_{m}\right]$ and $\rho_{m}\left(x+T_{m}\right)=\rho_{m}(x)$ for any $x \in \mathbb{R}$. Then $u_{m}$ is defined by the formula

$$
u_{m}(t)=\rho_{m}(t) \sin \left(2 \pi N_{m} t\right), \quad t \in \mathbb{R},
$$

where $N_{m}>N_{m-1}$ is a large integer to be determined later.

Now each $u_{m}$ is a periodic function on $\mathbf{R}, u_{m} \in L^{\infty}$, and none of the $u_{m}^{\prime}$ s overlap; that is, on any interval $[v, v+1], v \in \mathbb{Z}$, at most one $u_{m}$ is non-zero.

We put

$$
u(t)=\sum_{m=1}^{\infty} u_{m}(t), \quad t \in \mathbb{R} .
$$

The Poisson integral extends $u$ naturally to all of $P$.

LEMMA 1. The sequences $\left(T_{m}\right)_{m \geq 1}$ and $\left(N_{m}\right)_{m \geq 1}$ can be chosen so that $u$ is uniformly almost periodic on any line $L_{y}, y>0$.

Proof. Fix some line $L_{y_{0}}, y_{0}>0$. Since each $u_{m}$ is periodic on this line it is enough to show that the series defining $u$ in (3.1) converges uniformly on $L_{y_{0}}$.

We have

$$
u_{m}\left(x+i y_{0}\right)=\int_{-\infty}^{\infty} u_{m}(t) P^{\left(y_{0}\right)}(x-t) d t .
$$

Since the Poisson kernel consists of two monotonic pieces, we can apply Bonnet's form of the Second Mean Value Theorem to each piece. Thus

$$
\left|\int_{x}^{x+1} u_{m}(t) P^{\left(y_{0}\right)}(x-t) d t\right| \leq \frac{1}{\pi y_{0}} \cdot \frac{1}{\pi N_{m}},
$$

where we have estimated the integral 


$$
\left|\int_{x}^{x+1} u_{m}(t) d t\right|=\left|\int_{x}^{x+1} \rho_{m}(t) \sin \left(2 \pi N_{m} t\right) d t\right| \leq \frac{1}{\pi N_{m}}
$$

in the same fashion.

We now note that

$$
\left|\int_{x+1}^{\infty} u_{m}(t) P^{\left(y_{0}\right)}(x-t) d t\right| \leq \frac{1}{\pi} \sum_{N=1}^{\infty} \frac{y_{0}}{y_{0}^{2}+T_{m}^{2} N^{2}}
$$

since $\left|u_{m}(t)\right| \leq 1$, and that

$$
\begin{aligned}
\sum_{N=1}^{\infty} \frac{y_{0}}{y_{0}^{2}+T_{m}^{2} N^{2}} & \leq \frac{y_{0}}{y_{0}^{2}+T_{m}^{2}}+\int_{1}^{\infty} \frac{y_{0}}{y_{0}^{2}+T_{m}^{2} x^{2}} d x \\
& =\frac{y_{0}}{y_{0}^{2}+T_{m}^{2}}+\frac{y_{0}}{T_{m}} \int_{T_{m}}^{\infty} \frac{d s}{y_{0}^{2}+s^{2}} \\
& =\frac{y_{0}}{y_{0}^{2}+T_{m}^{2}}+\frac{1}{T_{m}} \int_{T_{m / y_{0}}}^{\infty} \frac{d v}{1+w^{2}} \\
& \leq \frac{y_{0}}{y_{0}^{2}+T_{m}^{2}}+\frac{y_{0}}{T_{m}^{2}} .
\end{aligned}
$$

Similar estimates can be given for the intervals $(x-1, x)$ and $(-\infty, x-1)$ so that we obtain

$$
\left|\int_{-\infty}^{\infty} u_{m}(t) P^{\left(y_{0}\right)}(x-t) d t\right| \leq \frac{2}{\pi^{2} y_{0} N_{m}}+\frac{2 y_{0}}{\pi}\left(\frac{1}{y_{0}^{2}+T_{m}^{2}}+\frac{1}{T_{m}^{2}}\right) \text {. }
$$

We now select the $N_{m}{ }^{\prime} s$ and the $T_{m}$ 's to satisfy both the conditions of the construction and the inequality

$$
\sum_{m=1}^{\infty}\left[\frac{2}{\pi^{2} y_{0} N_{m}}+\frac{2 y_{0}}{\pi}\left(\frac{1}{y_{0}^{2}+T_{m}^{2}}+\frac{1}{T_{m}^{2}}\right)\right]<\infty .
$$

This sum is independent of $x \in \mathbf{R}$ but will depend on the line $L_{y_{0}}$ chosen. With $\left(T_{m}\right)_{m \geq l}$ and $\left(N_{m}\right)_{m \geq l}$ thus chosen, $u$ is the uniform sum of periodic functions on the line $L_{y_{0}}, y_{0}>0$, and hence $u$ is almost 
periodic on any line in the upper half plane.

Since the sequence $\left(N_{m}\right)_{m \geq l}$ is increasing, we also have

LEMMA 2. The function $t \rightarrow u_{t}$ is not continuous from $\mathbb{R}$ into $\left(L^{1}, Z^{\infty}\right)$.

LEMMA 3. $u^{*} \in\left(L^{1}, 2^{\infty}\right)$.

Proof. This is trivial since $u \in L^{\infty}$.

LEMMA 4. The sequences $\left(T_{m}\right)_{m \geq 1}$ and $\left(N_{m}\right)_{m \geq 1}$ can be chosen so that $\tilde{u}$ is zoniformly almost periodic on any line $L_{y}, y>0$.

Proof. Note first of all that

$$
u(t)=\sum_{m=1}^{\infty} \tilde{u}_{m}(t), \quad t \in \mathbb{R}
$$

since the conjugation operator is continuous from $L^{\infty}$ into BMO and the series (3.1) defining $u$ converges absolutely. Fix some line $L_{y_{0}}$, $y_{0}>0 ;$ since each $\tilde{u}_{m}$ is periodic on this line (Proposition 1 ) it is enough to show that

$$
\sum_{m=1}^{\infty} \tilde{u}_{m}\left(x+i y_{0}\right)
$$

converges uniformly in $x \in \mathbb{R}$.

We begin by studying the boundedness of

$$
u_{m}(t)=\left[\rho_{m}(t) \sin \left(2 \pi N_{m} t\right)\right]^{-}, t \in \mathbb{R} .
$$

Since each $u_{m}$ is periodic on $\mathbb{R}$ we can consider it as a function on $\mathbf{T}$ by writing $g_{m}(t)=u_{m}\left(T_{m} t / 2 \pi\right), 0 \leq t<2 \pi$. Then we have $[6, p .55]$, 


$$
\begin{aligned}
\mid\left[\rho_{m}\left(\frac{x T^{m}}{2 \pi}\right) \sin \left(T_{m} N_{m} x\right)\right]^{\sim}-\rho_{m}\left(\frac{x T_{m}}{2 \pi}\right) & \left|\sin \left(T_{m} N_{m} x\right)\right|^{\sim} \mid \\
& =\left|P \cdot V \cdot \int_{0}^{2 \pi} \frac{\rho_{m}\left(t T_{m} / 2 \pi\right)-\rho_{m}\left(x T_{m} / 2 \pi\right)}{\tan ((t-x) / 2)} \sin \left(T_{m} N_{m} t\right) d t\right| \\
& \leq \frac{K T}{2 \pi}\left\|\rho^{\prime}\right\|_{\infty} \cdot \frac{1}{T_{m} N_{m}} \\
& =K\left\|\rho^{\prime}\right\|_{\infty} / 2 w_{m} .
\end{aligned}
$$

To obtain this estimate we have used the Mean Value Theorem applied separately to each of the monotonic pieces of the kernel

$$
\frac{\rho_{m}\left(t T_{m} / 2 \pi\right)-\rho_{m}\left(x T_{m} / 2 \pi\right)}{\tan ((t-x) / 2)}
$$

and the fact that this kernel is bounded by $T_{m}\left\|\rho^{\prime}\right\|_{\infty} / 2$. The constant $K$ is an upper bound for the number of monotonic pieces of the kernel and is independent of $m$.

Transferring back to $\mathbb{R}$ we have

$$
\tilde{u}_{m}(t)=\rho_{m}(t) \cos \left(2 \pi N_{m} t\right)+h_{m}(t), t \in \mathbb{R},
$$

where

$$
\left|h_{m}(t)\right|<K\left\|\rho^{\prime}\right\|_{\infty} / 2 N_{m}, \quad t \in \mathbb{R}
$$

and hence

$$
\left|\tilde{u}_{m}(t)\right| \leq 1+K\left\|\rho^{\prime}\right\|_{\infty} / 2 N_{m}, \quad t \in \mathbb{R}
$$

From this we see that if $I$ is any interval of unit length in $\mathbb{R}$ we have

$$
\begin{aligned}
\left|\int_{I} \tilde{u}_{m}(t) d t\right| & =\left|\int_{I}\left[\rho_{m}(t) \cos \left(2 \pi N_{m} t\right)+h_{m}(t)\right] d t\right| \\
& \leq \frac{1}{\pi N_{m}}+\frac{K\left\|\rho^{\prime}\right\|_{\infty}}{2 N_{m}},
\end{aligned}
$$

where again we have used the Mean Value Theorem applied separately to the (at most) two monotonic pieces of $\rho_{m}(t)$ over the interval $I$.

We now write 


$$
\tilde{u}_{m}\left(x+i y_{0}\right)=\int_{-\infty}^{\infty} \tilde{u}_{m}(t) P\left(y_{0}\right)(x-t) d t
$$

and argue as in Lemma 1 . We have

$$
\left|\int_{x}^{x+1} \tilde{u}_{m}(t) P^{\left(y_{0}\right)}(x-t) d t\right| \leq \frac{1}{\pi y_{0}}\left(\frac{1}{\pi N_{m}}+\frac{K\left\|\rho^{\prime}\right\|_{\infty}}{2 N_{m}}\right),
$$

and by $(3.4)$,

$$
\left|\int_{x+1}^{\infty} \tilde{u}_{m}(t) P^{\left(y_{0}\right)}(x-t) d t\right| \leq\left(1+\frac{K\left\|\rho^{\prime}\right\|_{\infty}}{2 N_{m}}\right) \frac{y_{0}}{\pi}\left(\frac{1}{y_{0}^{2}+T_{m}^{2}}+\frac{1}{T_{m}^{2}}\right) .
$$

Similar estimates hold over the intervals $(x-1, x)$ and $(-\infty, x-1)$ so that finally

$$
\begin{aligned}
\left|\int_{-\infty}^{\infty} \tilde{u}_{m}(t) P^{\left(y_{0}\right)}(x-t) d t\right| & \\
& \leq \frac{2}{\pi y_{0}}\left(\frac{1}{\pi N_{m}}+\frac{K\left\|\rho^{\prime}\right\|_{\infty}}{2 N_{m}}\right)+\frac{2 y_{0}}{\pi}\left(1+\frac{K\left\|\rho^{\prime}\right\|_{\infty}}{2 N_{m}}\right)\left(\frac{1}{y_{0}^{2}+T_{m}^{2}}+\frac{1}{T_{m}^{2}}\right) .
\end{aligned}
$$

We now select the $N_{m}^{\prime}$ s and $T_{m}$ 's to satisfy all previous conditions as well as

$$
\sum_{m=1}^{\infty} \frac{2}{\pi y_{0}}\left(\frac{1}{\pi N_{m}}+\frac{K \rho^{\prime} \infty_{\infty}}{N_{m}}\right)+\frac{2 y_{0}}{\pi}\left(1+\frac{K \rho^{\prime} \infty}{2 N_{m}}\right)\left(\frac{1}{y_{0}^{2}+T_{m}^{2}}+\frac{1}{T_{m}^{2}}\right)<\infty .
$$

This sum is independent of $x \in \mathbb{R}$ but will depend on the line $L_{y_{0}}$ chosen. With $\left(T_{m}\right)_{m \geq 1}$ and $\left(N_{m}\right)_{m \geq 1}$ thus chosen, $\tilde{u}$ is the uniform sum of periodic functions on the line $L_{y_{0}}$, and hence is uniformly almost periodic on any line in the upper half plane. This completes the proof.

In conclusion, the function $f=u+i \tilde{u}$ belongs to $H^{\mathcal{H}}$ but does not

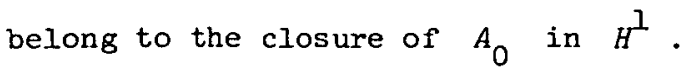




\section{References}

[1] J. Garnett, Bownded analytic finctions (Academic Press, New York and London, 1980).

[2] K. Hoffman, "Boundary behaviour of generalized analytic functions", Trans. Amer. Math. Soc. 87 (1958), 447-466.

[3] F. Holland, "Harmonic analysis on amalgams of $L^{p}$ and $\tau^{q} ", J$. London Math. Soc. (2) 10 (1975), 295-305.

[4] J. Milaszevitch, "Hardy spaces of almost periodic functions", Ann. Scuola Norm. Sup. Pisa Cl. Sci. (4) 24 (1970), 401-428.

[5] R. Owens, "A maximal function characterization of a class of Hardy spaces", submitted.

[6] A. Zygmund, Trigonometrical series, 2nd edition (Cambridge University Press, Cambridge, 1959).

Department of Mathematics, University of Western Australia,

Nedlands,

Western Australia 6009,

Australia. 